\title{
Proton radiography of dynamic electric and magnetic fields in laser-produced high- energy-density plasmas
}

\author{
C. K. Li ${ }^{1 *}$, F. H. Séguin ${ }^{1}$, J. A. Frenje ${ }^{1}$, M. Manuel ${ }^{1}$, D. Casey ${ }^{1}$, N. \\ Sinenian $^{1}$, R. D. Petrasso ${ }^{1}$, P. A. Amendt ${ }^{2}$, O. L. Landen ${ }^{2}$, J. R. Rygg ${ }^{2}$, \\ R. P. J. Town ${ }^{2}$, R. Betti ${ }^{3,4}$, J. Delettrez ${ }^{3}$, J. P. Knauer ${ }^{3}$, F. Marshall ${ }^{3}$, \\ D. D. Meyerhofer ${ }^{3,4}$, T.C. Sangster ${ }^{3}$, D. Shvarts ${ }^{3,5}$, V. A. Smalyuk ${ }^{3}$, J. \\ M. Soures ${ }^{3}$, C. A. Back ${ }^{6}$, D. Kilkenny ${ }^{6}$, and A. Nikroo ${ }^{6}$ \\ ${ }^{1}$ Plasma Science and Fusion Center, Massachusetts Institute of Technology, \\ Cambridge, Massachusetts 02139, USA \\ ${ }^{2}$ Lawrence Livermore National Laboratory, Livermore, California 94550 USA \\ 3 Laboratory for Laser Energetics, University of Rochester, Rochester, New York \\ 14623, USA \\ ${ }^{4}$ Department of Mechanical Engineering, Physics and Astronomy, University of \\ Rochester, Rochester, New York 14623, USA \\ ${ }^{5}$ NRCN, Negev and Ben Gurion University of the Negev, Beer-Sheva 84015, Israel \\ ${ }^{6}$ General Atomics, San Diego, California 92186 USA \\ * li@psfc.mit.edu
}

1 November 2008

Plasma Science and Fusion Center

Massachusetts Institute of Technology

Cambridge, MA 02139 USA

The work described here was performed in part at the LLE National Laser User's Facility (NLUF), and was supported in part by US DOE (Grant No. DE-FG03-03SF22691), LLNL (subcontract Grant No. B504974), and LLE (subcontract Grant No. 412160-001G).

Submitted to Physics of Plasmas 


\title{
Proton radiography of dynamic electric and magnetic fields in laser-produced high-energy-density plasmas
}

\author{
C. K. Li ${ }^{1 *}$, F. H. Séguin ${ }^{1}$, J. A. Frenje ${ }^{1}$, M. Manuel ${ }^{1}$, D. Casey ${ }^{1}$, N. Sinenian ${ }^{1}$, R. D. Petrasso ${ }^{1}$, \\ P. A. Amendt ${ }^{2}$, O. L. Landen ${ }^{2}$, J. R. Rygg ${ }^{2}$, R. P. J. Town ${ }^{2}$, R. Betti ${ }^{3,4}$, J. Delettrez ${ }^{3}$, J. P. Knauer $^{3}$, \\ F. Marshall $^{3}$, D. D. Meyerhofer ${ }^{3,4}$, T.C. Sangster ${ }^{3}$, D. Shvarts ${ }^{3,5}$, V. A. Smalyuk ${ }^{3}$, J. M. Soures ${ }^{3}$, \\ C. A. Back ${ }^{6}$, D. Kilkenny ${ }^{6}$, and A. Nikroo ${ }^{6}$ \\ ${ }^{1}$ Plasma Science and Fusion Center, Massachusetts Institute of Technology, Cambridge, Massachusetts 02139, USA \\ 2 Lawrence Livermore National Laboratory, Livermore, California 94550 USA \\ ${ }^{3}$ Laboratory for Laser Energetics, University of Rochester, Rochester, New York 14623, USA \\ ${ }^{4}$ Department of Mechanical Engineering, Physics and Astronomy, University of Rochester, Rochester, New York 14623, USA \\ ${ }^{5}$ NRCN, Negev and Ben Gurion University of the Negev, Beer-Sheva 84015, Israel \\ ${ }^{6}$ General Atomics, San Diego, California 92186 USA \\ * li@psfc.mit.edu
}

Time-gated, monoenergetic-proton radiography provides unique measurements of the electric $(E)$ and magnetic $(B)$ fields associated with laser-foil interactions, imploded inertial-confinement-fusion capsules, and laser-irradiated hohlraums. These experiments resulted in the first observations of several new and important features previously unrealized: first, observations of the generation, decay dynamics, instabilities and topology change of megagauss $B$ fields on laser-driven planar plastic foil; second, the observation of radial $E$ fields inside the imploding capsule which are initially directed inward, reverse direction near deceleration onset, and are likely related to the evolution of the electron pressure gradient; third, the observation of many radial filaments with complex electromagnetic field striations, permeating the entire field of view, and fourth, the observation of dynamic $E$ field associated with laser-irradiated gold hohlraums. The physics behind and implications of such observed fields are discussed.

\section{INTRODUCTION}

The generation, evolution, interaction, and dissipation of self-generated electric $(E)$ and magnetic $(B)$ fields by interactions of laser light with matter are profound processes that are of fundamental interest in High-Energy-Density (HED) physics (pressures >1 megabar) ${ }^{1}$, and which are attracting increasing attention. The primary mechanism behind field formation is the loss of energetic electrons from the heated region, resulting in the breakdown of charge neutrality. Many processes contribute to field generation and evolution, but their relative importance depends on interaction parameters ${ }^{1-8}$. For long-pulse, low-intensity laser light, as illustrated in Fig. 1, the dominant source for $B$ field generation are noncollinear electron density and temperature gradients $\left(\nabla n_{e} \times \nabla T_{e}\right)$. In particular, for circular laser spots, the $B$ fields have a toroidal configuration with scale length comparable to the spot size. In the regime with low $Z$ and high temperature, where resistivity is low, $B$-field growth is usually linear in time and is balanced primarily by convective losses $[\nabla \times(\boldsymbol{v} \times \mathbf{B})$, where $\boldsymbol{v}$ is the plasma fluid velocity; i.e., the $B$ field is "frozen in"]. When the laser is off and the cooling plasma becomes more resistive, field diffusion dominates convective transport $\left[\nabla \times\left(D_{m} \nabla \times \mathbf{B}\right)\right.$, where $D_{m}$ is the magnetic

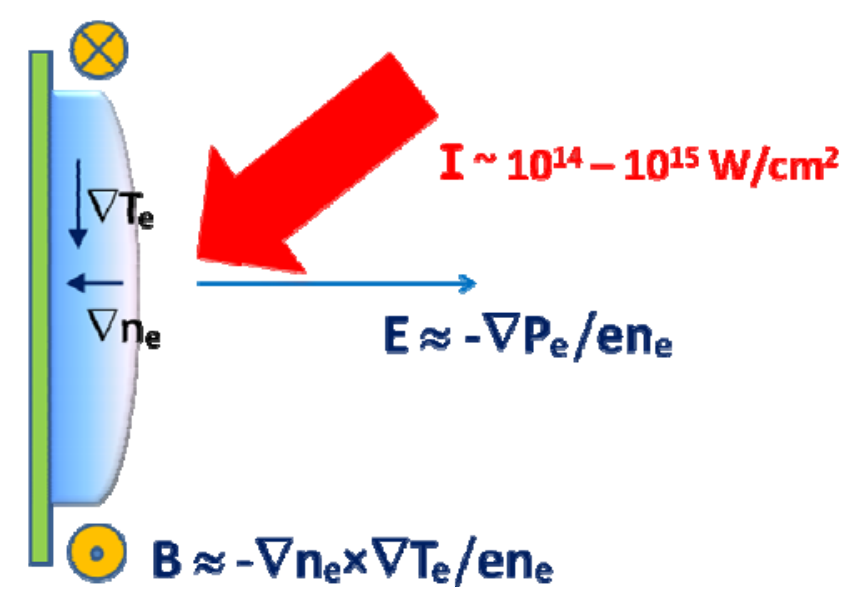

FIG.1 (Color) Schematic illustration of the generation of self-generated $E$ and $B$ fields by laser-matter interactions, described by the Faraday equation (Eq. 1) combined with a simplified version of the generalized Ohm's law ${ }^{1-8}$. 
diffusion coefficient]. Under these circumstances, $B$ field generation and evolution are

$$
\frac{\partial \mathbf{B}}{\partial \mathrm{t}} \approx \nabla \times(\mathrm{v} \times \mathbf{B})-\frac{1}{\mathrm{en}_{\mathrm{e}}} \nabla \mathrm{n}_{\mathrm{e}} \times \nabla \mathrm{T}_{\mathrm{e}}-\nabla \times\left(\mathrm{D}_{\mathrm{m}} \nabla \times \mathbf{B}\right) .
$$

The dominant source for $E$ fields is the electron pressure gradient $\left(\nabla P_{e} / n_{e}\right)$, a consequence of force balance in a single fluid force equation (where electron inertial is neglected) ${ }^{1-4}$,

$$
0 \approx \mathbf{E}-\frac{1}{\mathrm{en}_{\mathrm{e}}} \nabla \mathrm{P}_{\mathrm{e}}
$$

In addition to their critical importance to fundamental HED physics, these fields have important implications for several current problems. For example, in inertial-confinement fusion (ICF) ${ }^{1,9-11}, B$ fields generated inside a hohlraum by laser illumination can reduce heat flow, since cross-field thermal conductivity is modified by a factor of $\left(1+\omega_{c e}{ }^{2} \tau^{2}\right)^{-1}$, where $\omega_{c e}$ is the electron gyro frequency and $\tau$ is the collision time. The generation of $E$ field, on the other hand, may significantly modify the plasma distribution and enhance the thick-target bremsstrahlung. The effects of these fields are the altered distributions of electron temperature and density, which lead to significantly enhanced laser-plasma instabilities, radiation nonuniformity, and background emissions ${ }^{1,9-11}$.

We have developed the new method of monoenergetic, charged-particle radiography ${ }^{6-8,12}$ and used it in new types of studies of $E$ and $B$ fields, as well as plasmas in HEDP and ICF physics. The experiments are performed at the OMEGA laser facility ${ }^{13}$. Topics we study include generation, time evolution and instabilities in laser-plasma-generated $B$ fields, reconnection of MG magnetic fields in high- $\beta$ plasmas, self-generated $E$ and $B$ fields in direct-drive ICF implosions, $B$ fields associated with Rayleigh-Talyor instabilities in laser-driven foils, and dynamic $E$ and $B$ fields in laser-driven hohlraums. Our work has resulted so far in several important observations that have never before been observed ${ }^{6-8,12,14,15}$. In addition, this work has successfully addressed both basic physics issues and those directly relevant to the future success of ignition experiments at the National Ignition Facility $(\mathrm{NIF})^{10}$.

In this article, the progress made in recent studies of $E$ and $B$ fields using proton radiography is summarized. Section II briefly describes the technology of proton radiography and the experiments. The experimental results and discussions are presented in section III. We summarize the important findings and results in Section IV.

\section{PROTON RADIOGRAPHY OF FIELDS GENERATED BY LASER-PLASMA INTERACTIONS}

\section{Ila. A monoenergetic proton backlighter}

To quantitatively probe laser-plasma interactions, and particularly resultant $E$ and $B$ fields, an imaging technology that combines a monoenergetic-particle backlighter with a matched detection system ${ }^{6-8,12,14-17}$ has been developed. This approach has distinct advantages over radiography with broad-band proton sources (such as intense-laser-induced sources). The monoenergetic particles are nuclear fusion products resulting from $\mathrm{D}^{3} \mathrm{He}$-filled, exploding-pusher implosions at OMEGA. 14.7-MeV protons, $3-\mathrm{MeV}$ protons, and 3.6-MeV alphas are generated from the nuclear reactions, as shown below.

$$
\begin{aligned}
& \mathrm{D}+{ }^{3} \mathrm{He} \rightarrow \alpha(3.6 \mathrm{MeV})+\mathrm{p}(14.7 \mathrm{MeV}) \\
& \mathrm{D}+\mathrm{D} \rightarrow \mathrm{T}(1 \mathrm{MeV})+\mathrm{p}(3 \mathrm{MeV})
\end{aligned}
$$

Backlighter implosions are typically driven with 20 or fewer OMEGA laser beams (wavelength of $0.351 \mu \mathrm{m}$ ) 13 in either a 8 to $10 \mathrm{~kJ}, 1-\mathrm{ns}$ square pulse or a 2.5 to 5 $\mathrm{kJ}$, 0.6-ns pulse; no phase plates or smoothing by spectral dispersion $(\mathrm{SSD})^{18-20}$ are used. The capsule diameter is small, at about $440 \mu \mathrm{m}$, in order to yield a smaller-than-usual burn radius for improved spatial resolution in the radiograph. A comprehensive set of diagnostics are used to characterize the implosion, including both proton-emission and $\mathrm{x}$-ray-emission images for studying the size of the imploded capsule and its burn region, spectrometers for measuring the proton energy spectrum ${ }^{12,16,17}$, and a proton temporal diagnostic for measuring the bang time and burn duration $^{12,21}$. Each fusion product is monoenergetic (Fig. 2a) and produced only during a 130-ps time interval (Fig. 2b). The size of the emitting region is measured $^{6-8,12,14-17}$; to be about $40 \mu \mathrm{m}, \mathrm{FWHM}$. The relative timing of the primary and backlighter implosions is adjusted so that the radiographic image records the condition of the laser-induced plasma at two different times relative to the arrival of the interaction beam, to provide coarse time evolution information. 

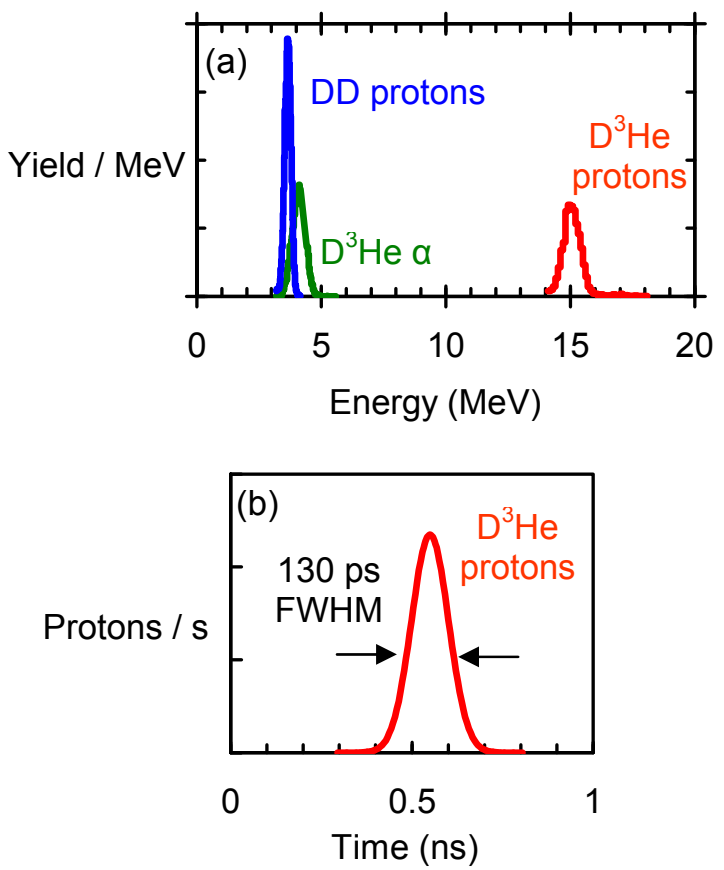

FIG. 2. (Color online) Spectra (a) and time history (b) of charged fusion products from an exploding pusher target at OMEGA containing $\mathrm{D}^{3} \mathrm{He}$ fuel. Note that the particle energies are slightly upshifted from their birth energies, due to the charging of the capsule during the laser illumination $^{12,16,17,22}$.

\section{Ilb. Proton radiography of laser-produced high-energy-density plasmas}

Backlighters are used in simple radiography configurations as shown in Fig. 3, although their isotropic character leads us to backlight at least two different subjects at the same time, at different angles relative to the backlighter, in order to maximize the amount of data that can be accumulated during a given number of shots. The proton-flux images are captured through CR-39 nuclear accuracy by automated scanning systems. The incident energy of each individual proton is also determined from its track in the CR-39. Detection efficiency is essentially $100 \%$. After the detectors are etched in $\mathrm{NaOH}$, the location of incidence of each individual proton is identified and measured to a submicron ${ }^{12}$.

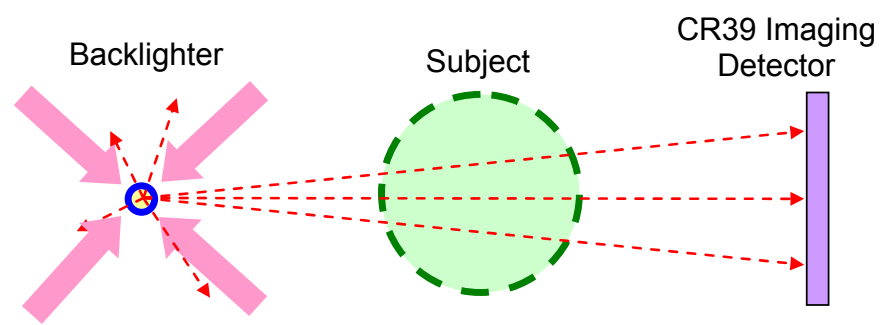

FIG. 3. (Color online) Basic configuration of the monoenergetic charged particle radiography setup. Sample subjects are shown in Fig. 4. In some cases, a single backlighter is used to image two subjects located at 90 or 180 degrees relative to the backlighter; each subject has its own detector. Typical system dimensions are $1 \mathrm{~cm}$ from backlighter to subject, and $30 \mathrm{~cm}$ from backlighter to detector.

Some of the subjects radiographed are shown in Fig. 4, and will be discussed in the following subsections. The monoenergetic character of the particles used for imaging allows us to simultaneously make two kinds of measurements of the imaged subject. First, the energy of the particle indicates how much energy the particle lost while passing through the subject, allowing the areal density of the subject to be studied. Second, deflections of the trajectories of the particles by $B$ or $E$ fields can be measured, and used to quantitatively study field strengths. In some experiments, the measurement of particle deflection is made easier by the insertion of a metal mesh between the backlighter and the subject, as indicated in Figs. 4a, $4 \mathrm{~b}, 4 \mathrm{c}$, and $4 \mathrm{~g}$; the mesh divides the protons into beamlets whose individual positions can be measured with great accuracy at the detector.

Figure 5 shows how a single plasma bubble, formed by the interaction of a laser beam with a plastic $(\mathrm{CH})$ foil, is surrounded by a toroidal magnetic field that deflects individual proton beamlets to form a well defined image, the individual positions of which can be compared on the detector with the positions they would have had in the absence of the magnetic field ${ }^{6-8,12,15}$. The identification of the field as $B$ rather than $E$ is demonstrated unambiguously ${ }^{6,12,24}$, taken from an image obtained with the subject shown in Fig. $4 b^{24}$. 
(a)

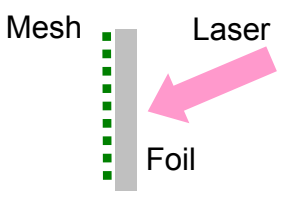

Laser on foil with mesh

(e)

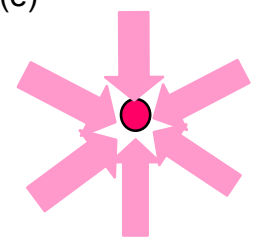

Laser on

Spherical capsule (b)

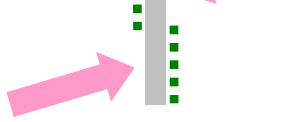

"Double-Bubble" experiment

(f)

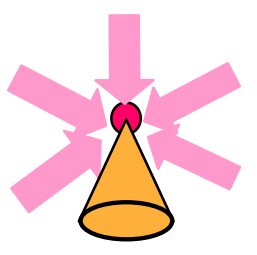

Laser on Cone-In-Shell ICF capsule (c)

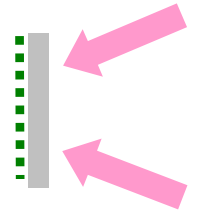

Multiple laser beams on foil with mesh (d)

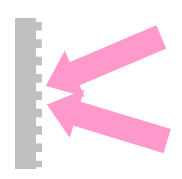

Laser on rippled "Rayleigh-Taylor" foil

(g)

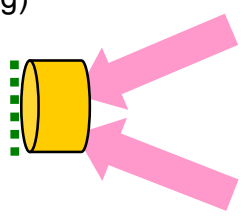

Laser on

hohlraum with mesh

FIG. 4. (Color online) Some of the subjects used in radiography experiments so far, with the configuration shown in Fig. 3 and the imaging particles incident from the left. The images that result from subjects (a) - (d), with the particles placed normally in relation to the foil, are referred to as "face-on", because they show structure in the plane of the foil. Images of these subjects are also recorded with the hohlraum oriented parallel to the imaging particles, showing the "end-on" structure.

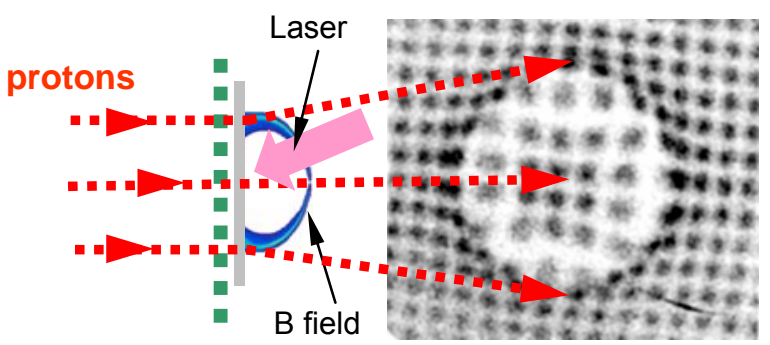

FIG. 5. (Color) Illustration of a laser-plasma interaction on a $\mathrm{CH}$ foil, together with an actual radiography image formed by $15-\mathrm{MeV}$ protons divided into beamlets by a metal mesh (the darker areas indicate more protons $)^{6}$. LASNEX+LSP simulations ${ }^{25-}$ 28 indicate that face-on radiography will be sensitive only to the $B$ field, while side-on radiography will be sensitive only to the $E$ field. This allows $E$ and $B$ fields to be measured separately.

\section{DATA AND DISCUSSIONS}

\section{IIla. The generation, time evolution and instabilities of $B$ fields on laser-driven $\mathrm{CH}$ foils}

Proton radiography was used to make the first measurements of the long-timescale dynamics and evolution of megagauss laser-plasma-generated magnetic field structures, as shown in Fig. 6. The monoenergetic character of the proton source, together with the well-characterized imaging detectors and geometry, make quantitative analysis of the images possible. The typical field strength was estimated $\sim 10^{6}$ gauss $(\sim 1 \mathrm{MG})^{6-8}$. Fig. 7 shows the time evolutions of the bubble asymmetry and the maximum value of $\left|\int \mathbf{B} \times \mathrm{d} \boldsymbol{\ell}\right|$. The images show that, while a 1-ns $10^{14}$ $\mathrm{W} / \mathrm{cm}^{2}$ laser beam is on, the field structure expands in tandem with a hemispherical plasma bubble, maintaining a rigorous two-dimensional (2D) cylindrical symmetry (see also Fig. 5). With the laser off, the bubble continues to expand as the field decays; however the outer field structure becomes distinctly asymmetric, indicating instability. Similarly, localized asymmetry growth in the bubble interior indicates another kind of instability ${ }^{7,29}$. 


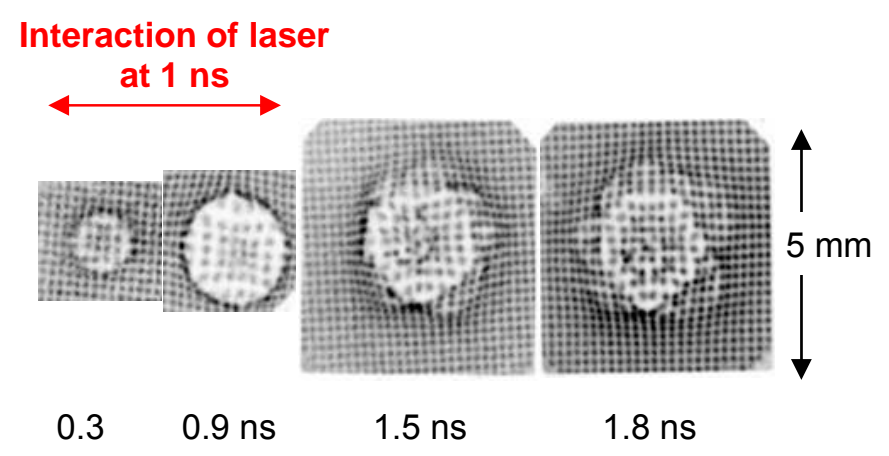

FIG. 6. $\mathrm{D}^{3} \mathrm{He}$ proton radiographs showing the evolution of B fields in a plasma/field bubble formed by the interaction of a laser beam with a $\mathrm{CH}$ foil, as described in the text and Ref. 7. The arrangement of the foil with respect to the imaging system is shown in Figs. 2 and 3a.

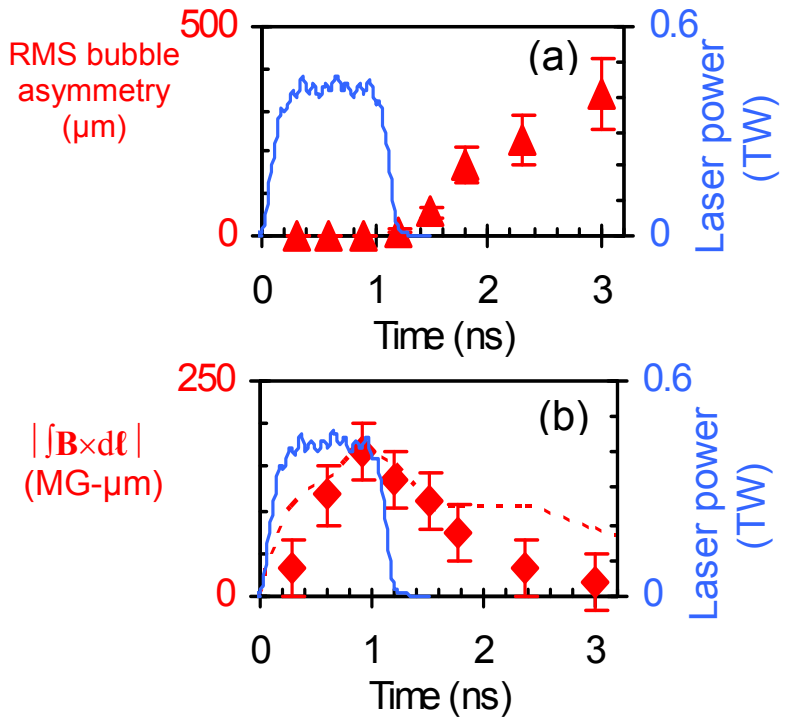

FIG. 7. (Color online) Measured time evolution of RMS deviations of the outer bubble boundary from the average radii (a), and maximum $\left|\int \mathbf{B} \times \mathrm{d} \boldsymbol{\ell}\right|$ (b), as described in Ref. 7. In each case, the blue curve is the time history of the laser intensity, and the red dashed line shows the result of a 2D LASNEX+LSP simulation (Fig. 9).

This is the first observation of such an instability in laser-produced HED plasmas. As is illustrated in
Fig. 8, it is plausible that this is a pressure-driven, resistive magnetohydrodynamic (MHD) interchange instability $^{7}$, resulting in an interchange of field between the inside and outside of the bubble surface. It occurs under circumstances such as we have here, with unfavorable field curvature $(\boldsymbol{\kappa} \cdot \nabla p>0$, where $\boldsymbol{\kappa}$ $=\mathbf{B} \cdot \nabla \mathbf{B} / \mathrm{B}^{2}$ is the field-line curvature and $\nabla p$ is the pressure gradient) and when resistivity allows for radial diffusion of $B$ field into the region outside the bubble. It makes sense that this instability would occur only after the laser is off, as shown in Fig. 7a, when the cooling plasma becomes more resistive. Of course, we cannot, without additional experiments, completely rule out the possible role of other MHD instabilities, (such as kink or tearing modes ${ }^{30}$,) but those seem less likely to have the observed spatial configuration. Pure fluid instabilities such as the Widnall type ${ }^{31}$, on the other hand, might be expected to be visible while the laser is on (when $B$ fields don't have much impact on the flow but are frozen in); but we don't see any evidence for the instability occurring then ${ }^{7}$.

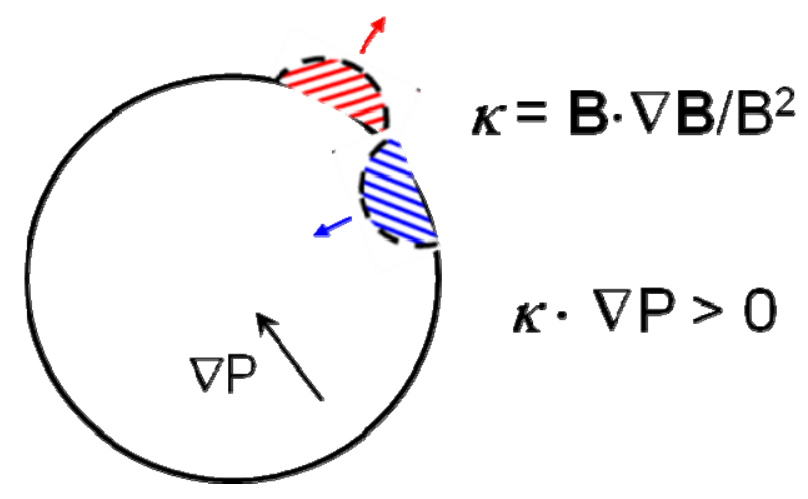

FIG. 8. (Color online) Illustration of a pressuredriven, resistive MHD interchange instability resulting in an interchange of field between the inside and outside of the bubble surface. 


\section{IIlb. Evaluation of 2D modeling of laser- plasma interactions; the importance of 3D simulations}

Two dimensional hydrodynamic simulations have been carried out for several of these experiments using the code LASNEX ${ }^{25,26}$ for the magnetic field package and $\mathrm{LSP}^{27}$ for proton transport. As an example, Fig. 9 shows the result of post-processing simulations for the experiments, which resulted in the data of Fig. 6 and produced simulated proton radiographs. The net result of the data-to-simulation comparison is that the $2 \mathrm{D}$ code does a reasonable job of predicting the cylindrically "averaged" 2D behavior of the bubble, (and B field generation and structure of the asymmetry seen in the

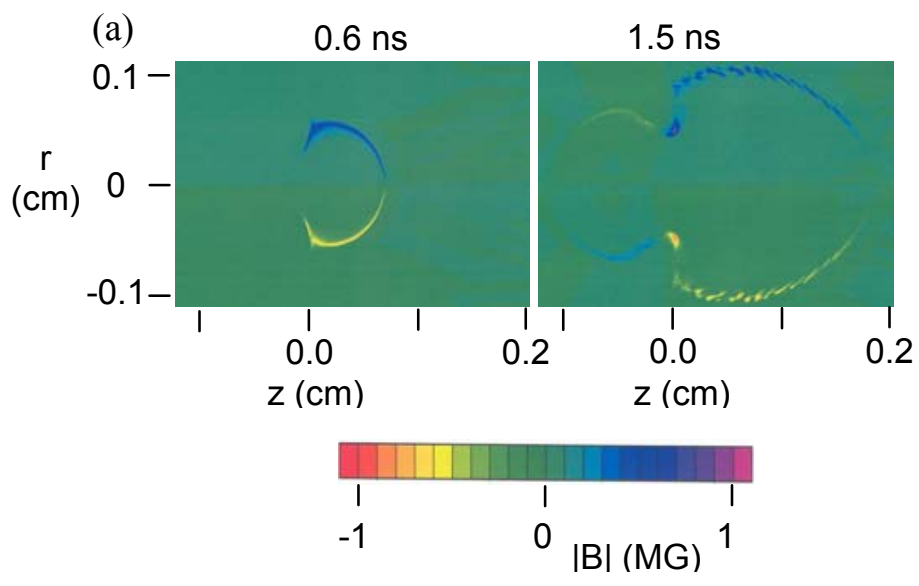

(b)
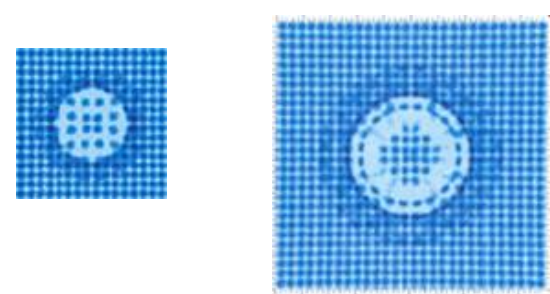

FIG. 9 (Color) LASNEX-simulated $B$ field strength (a) on cross sections of the bubble in a plane perpendicular to the foil at $\sim 0.6 \mathrm{~ns}$, when laser was on, and $\sim 1.5 \mathrm{~ns}$, when laser was off. The horizontal coordinate $z$ is distance from the foil (the laser is incident from the right and the protons travel from left to right) and the vertical coordinate $r$ is distance from the central axis of the plasma bubble. (b) Images generated from 2D LASNEX+LSP simulations of the laser-plasma interactions. data at late expansion, ) but underestimates the rate of field dissipation and of course doesn't predict the 3D times. Experimental measurements such as those shown here (and in the next section) are important because they directly reveal previously unpredictable physical phenomena, indicate the fundamental importance of 3D processes in certain regimes (such as in this decay phase) and provide invaluable information for benchmarking true 3D code development in the future. Now that the 3D hydrocode HYDRA with a field generating package ${ }^{32}$ is available, important comparisons between observations and simulations can be made in the future.

\section{IIIc. Magnetic reconnection in laser- generated plasmas}

The interaction and reconnection of magnetic fields in plasmas are important fundamental processes ${ }^{33}$ with implications for a wide range of basic sciences, including astrophysics ${ }^{34}$, space physics $^{35}$, and laboratory physics ${ }^{36,37}$, In the frontier field of HEDP (pressures $>1$ megabar) ${ }^{1}$, the generation, evolution, and reconnection of $B$ fields due to laser-plasma interactions takes place in an extreme physical regime. High plasma densities $\left(>10^{20} \mathrm{~cm}^{-3}\right)$, high temperatures $(\sim 1 \mathrm{keV})$, intense B fields $(\sim 1 \mathrm{MG})$, and high ratios of thermal pressure to magnetic pressure $(\beta>>1)$ distinguish this novel regime from tenuous plasmas, of order $10^{14} \mathrm{~cm}^{-3}$ or (usually much) lower, which are the more traditional venues of reconnection experiments ${ }^{33,36,37}$. Radiography experiments described in Ref. 8 involved the first direct observation of field reconnection in the HED regime, where plasma flow was dominated by hydrodynamics and wasn't strongly affected by fields, even though MG fields were present. The setup was as shown in Figs. 3 and 4c, with two laser beams on a foil. The results, shown in Fig. 10, have fundamental implications for basic reconnection physics in all regimes. Quantitative field maps derived from the radiographs reveal precisely and directly, for the first time, changes in the magnetic topology due to reconnection in a high-energy-density plasma $\left(n_{e} \sim\right.$ $\left.10^{20}-10^{22} \mathrm{~cm}^{-3}, T_{e} \sim 1 \mathrm{keV}\right)$.

These observations provide the first precise measurements and mapping of the change in field topology associated with reconnection in a high- $\beta$, HED experiment. Furthermore, this reconnection is intrinsically $3 \mathrm{D}$, and neither 2D LASNEX nor even the 
(a) $0.67 \mathrm{~ns}$

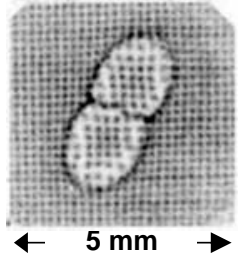

(b)

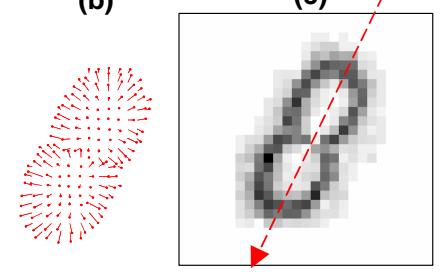

(d)

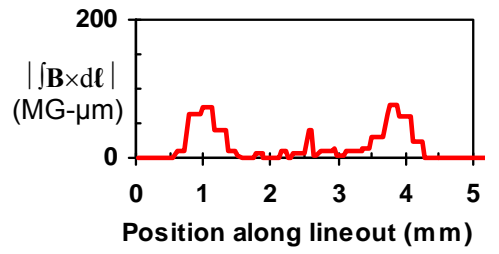

FIG. 10. (Color online) Radiographs generated when two laser beams were incident on a $\mathrm{CH}$ foil, showing how magnetic reconnection results in diminished field energy where two plasma bubbles surrounded by magnetic fields collide. The bubbles have interacted and reconnection has occurred by $0.67 \mathrm{~ns}$. At later times, most fields in the intersection region have reconnected (remaining distortion is largely due to burn-through holes.) The images were used to deduce maps of the $B$ field at the foil. In the 0.67-ns image, the location of each beamlet can be compared with the location it would have had without $B$ fields (beamlets on the image edges define the grid of "undeflected" locations); (b) shows displacement vectors $\xi$ of the beamlets. Arrays of displacement amplitudes are shown as images in (c); each pixel represents one beamlet, with a value proportional to displacement. Displacement is proportional to $\int \mathbf{B} \times \mathrm{d} \boldsymbol{\ell}$ along the particle trajectory, so lineouts of (c) (along the red arrow) provide quantitative measurement of $\left|\int \mathbf{B} \times \mathrm{d} \boldsymbol{\ell}\right|$ at the foil location (d).

2D Sweet-Parker model ${ }^{38,39}$ can be expected to adequately and quantitatively articulate such a process. The experimental time scale for reconnection can be estimated as $\tau_{R, E} \sim 0.2 \mathrm{~ns}$ by dividing the apparent width of the field layer at the surface of the bubble in Fig. 9c by twice the bubble expansion velocity. In contrast, the SP reconnection time is $\tau_{R, S P}=\left(\tau_{D} \tau_{A}\right)^{1 / 2} \sim 5$ ns, where $\tau_{D}=0.5 L_{\perp}{ }^{2} D_{m}{ }^{-1} \sim 30 \mathrm{~ns}$ is the $B$-field diffusion time, $\tau_{A}=L_{\perp} v_{A}{ }^{-1} \sim 1 \mathrm{~ns}$ is the Alfvén transit time, and $v_{A} \sim 5 \times 10^{6} \mathrm{~cm} \mathrm{~s}^{-1}$ (taking $n_{i} \sim 1 \times 10^{20} \mathrm{~cm}^{-3}$ and inferring from earlier results ${ }^{6,7}$, that $B \sim 0.5 \mathrm{MG}$ for a proton path length $\sim 200 \mu \mathrm{m})$. Though there are uncertainties in the estimates of these parameters, the comparison suggests that the reconnection illustrated in Fig. 10 is dominated by plasma hydrodynamics and noncollisional (nondissipative) processes, rather than resistivity. This dominance is connected with the fact that the plasma expansion velocity is faster than the Alfvén velocity. Unlike all previous reconnection experiments, the ones described here have a reconnection time shorter than the characteristic Alfvén transit time.

\section{IIId. Self-generated $E$ and $B$ fields in direct- drive ICF implosions}

As described in Refs. 14 and 15, radiography of inertial fusion implosions has resulted in the discovery and characterization of unexpected electromagnetic fields and in the measurement of the temporal evolution of capsule size and areal density. Figure 11 shows radiographs taken at different times during implosions of cone-in shell capsules for fast ignition ${ }^{40}$ (11a) and direct-drive spherical implosions ${ }^{41}(11 \mathrm{~b})$, using the setup of Fig. 2, with subjects oriented as shown in Figs. $3 \mathrm{f}$ and $3 \mathrm{e}$, respectively, and covering a large enough region outside the capsules to show external field structures. Figure 12 shows radiographs of the implosions of spherical capsules at many times during typical implosions, but displaying only the field of view including the capsule and its interior ${ }^{15}$. The fluence images contain information about both fields and matter, since fields deflect protons and matter scatters protons, but the signature of the compressed shell is in each case a circular feature with reduced proton fluence (as seen most clearly in the 0-ns images of Figs. 11a, and $11 \mathrm{~b}$ ), while the signature of the $\mathrm{Au}$ cone in the cone-in-shell capsules is a cone-shaped region with no proton fluence $^{14}$.

The images reveal several unexpected features due to electromagnetic fields in and around the imploding capsules. In the two images of Fig. 11a, the region outside the capsule shell is uniform at $0 \mathrm{~ns}$ but full of 
(a) Cone-in shell capsule

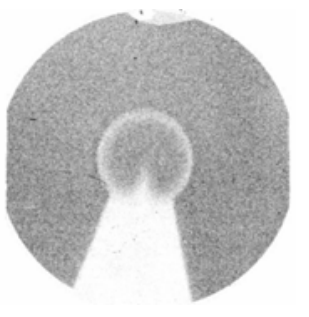

$0 \mathrm{~ns}$

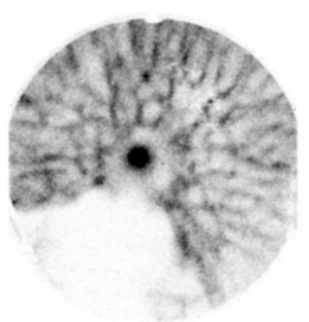

$1.5 \mathrm{~ns}$ (b) Spherical capsules

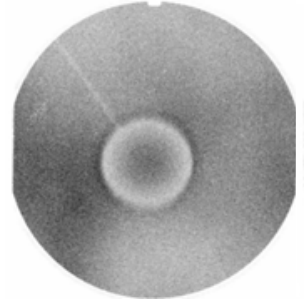

0 ns

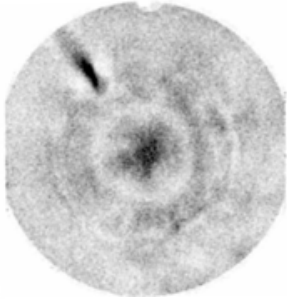

$0.5 \mathrm{~ns}$

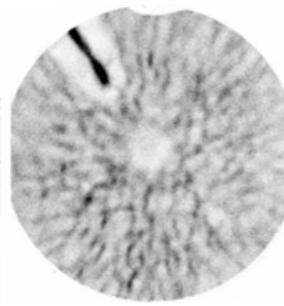

$1.5 \mathrm{~ns}$
FIG. 11. Proton fluence distributions in radiographs of (a) a 430- $\mu$ m-radius spherical plastic capsule with attached $\mathrm{Au}$ cone, before and during implosion ${ }^{14}$, and (b) spherical capsules before and during implosion 15,42 . In all cases, the 1 -ns-long laser drive began at 0 ns. The plastic shells were $24 \mu \mathrm{m}$ thick in (a), and 20 $\mu \mathrm{m}$ thick in (b). The linear feature in the upper left corner of each image of a spherical capsule is the mounting stalk.

filaments with complex bifurcations at $1.5 \mathrm{~ns}$. The deflections of imaging protons necessary to generate this filamentation cannot be explained due to scattering in filamented matter, which would require much more material than is present in the corona. The filamentation must be due to fields, and is qualitatively repeatable in different experiments, as seen in the 1.5-ns image of Fig. 11b. The precise nature and source of the fields responsible for these filaments is under study, but as magnetic fields they can be shown from the scale and amplitude of the features to have a characteristic magnitude of 60 tesla $^{14}$. The 0.5 -ns image in Fig. 11b shows that the field structure at earlier times has a different character, since the features outside the capsule have a quasi-spherical structure. The time evolution of these fields, and their transitions from one type to another, will be discussed elsewhere ${ }^{42}$. Although the mechanism for generating these fields is unclear, their effect on implosion dynamics is potentially consequential.
The figures also show the existence of another type of field inside the imploding capsules. The fluence images of Fig. 12 at 0.8 show a central peak in fluence. Monte Carlo simulations have shown that the size of this peak cannot be explained by the scattering of imaging protons by the $\mathrm{CH}$ shell. It must therefore be due to a radial $\mathrm{E}$ field directed inward (at $\sim 10^{9} \mathrm{~V} / \mathrm{m}$ ). The images show that the centrally peaked fluence turns into a central deficit of fluence at later times $(>1.4$ $\mathrm{ns})$, indicating that the $\mathrm{E}$ field reversed direction and has a magnitude of $\sim 10^{8} \mathrm{~V} / \mathrm{m}$. Such a field is a probable consequence of the evolution of the electron pressure gradient; this can be seen in Fig. 13, which shows that the experiment-implied $\mathrm{E}$ field magnitude at different times compares well with the field calculated from the pressure gradients predicted by the $1 \mathrm{D}$ hydrodynamic code LILAC $^{43}$. (Note that in Fig. 11b, where the capsule is of the same type as in Fig. 13, the central deficit has disappeared by $1.5 \mathrm{~ns}$, which is consistent with Fig. 12. In Fig. 11a, where the shell is thicker and the compression slower, the central peak persisted at least until $1.5 \mathrm{~ns}$.

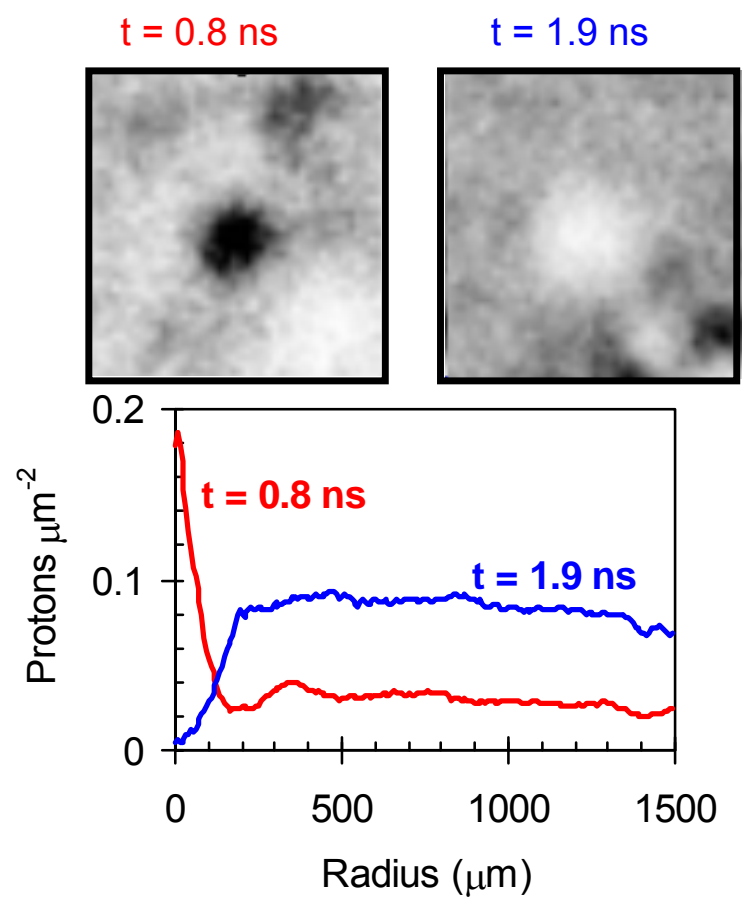

FIG. 12. (Color online) Radial profiles of the proton fluence images at $\mathrm{t}=0.8 \mathrm{~ns}$ and $1.9 \mathrm{~ns}$. Comparitively, a fluence peak occurs in the image centers during the early stages of implosion, indicating a "focusing" of imaging protons there, while in contrast, the fluence is extremely low, or defocused, at the image centers at later times. Note that the different level $(x \sim 2)$ of the proton fluence outside the capsules $(r>\sim 200 \mu \mathrm{m})$ is due to the variations from the backlighter proton yields. 


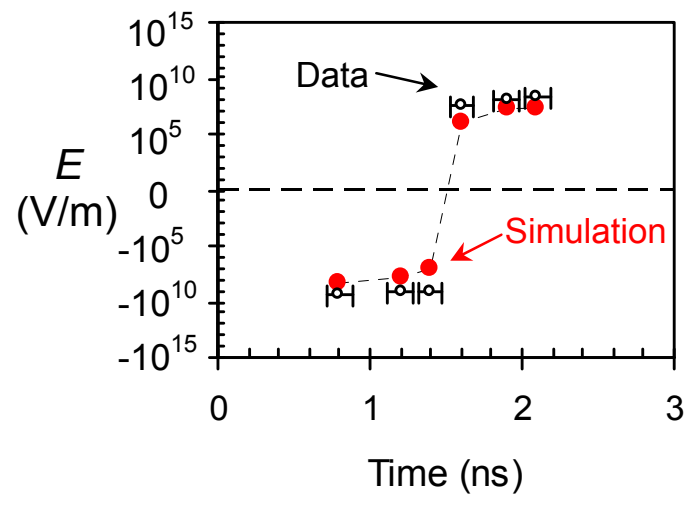

FIG. 13. (Color online) $15-\mathrm{MeV}$ proton radiographs of imploding capsules (20- $\mu \mathrm{m}$ shells) at different times, illustrating the time evolution of mass distribution and of a previously unobserved radial electric field ${ }^{15}$. In the fluence images (a), darker means higher fluence, while in the energy images (b) darker means lower proton energy (more matter traversed). The capsule-mounting stalk appears in the lower right corner of each fluence image.

\section{IIle. Electric fields associated with laser- driven gold hohlraums}

Radiography is also being used to probe dynamic $\mathrm{E}$ and $\mathrm{B}$ fields generated inside hohlraums when lasers impinge on their inner walls as shown in Fig. $3 \mathrm{~g}$. Ten laser beams irradiated in an incident angle of $58.8^{\circ}$ were used with an $\mathrm{Au}$ hohlraum that was $2.4 \mathrm{~mm}$ in diameter and $2 \mathrm{~mm}$ long. A mesh divided the imaging particles into beamlets, and radiographs were made using three different particles: $15-\mathrm{MeV} \mathrm{D}^{3} \mathrm{He}$ protons, $3-\mathrm{MeV}$ DD protons, and $3.6-\mathrm{MeV} \quad \mathrm{D}^{3} \mathrm{He}$ alpha particles. These experiments represent the first effort to find direct evidence of fields ${ }^{44}$ associated with laserhohlraum interactions. Such observations are very important because internal fields can modify the transport of electrons and energy, potentially having significant impact on the process of imploding an ICF capsule in a laser-driven hohlraum ${ }^{45}$. Figure 14 shows resulting images before the laser beams were incident, and $0.8 \mathrm{~ns}$ after the laser beams were incident, proving the feasibility of this important type of imaging. The image recorded with $3-\mathrm{MeV}$ protons at $0.8 \mathrm{~ns}$ shows that the trajectories of these low-energy protons were modified by a field inside the hohlraum reflecting the symmetry of the laser beams (the ten beams were actually arranged in five pairs, see Fig. 16).

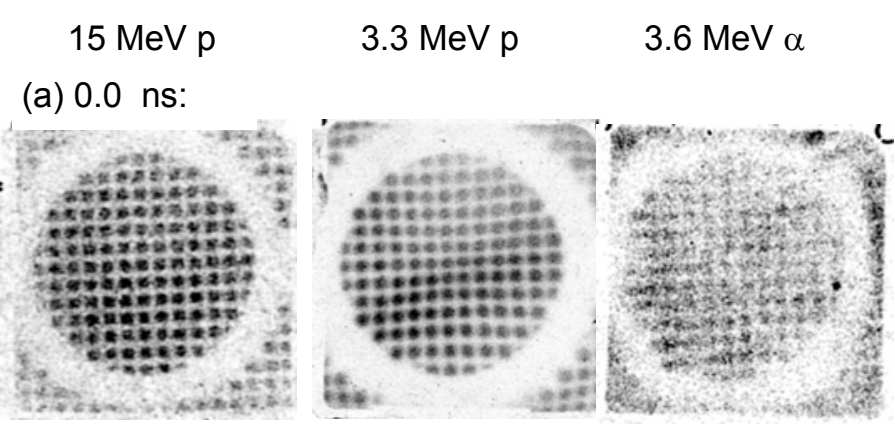

(b) 0.8 ns:

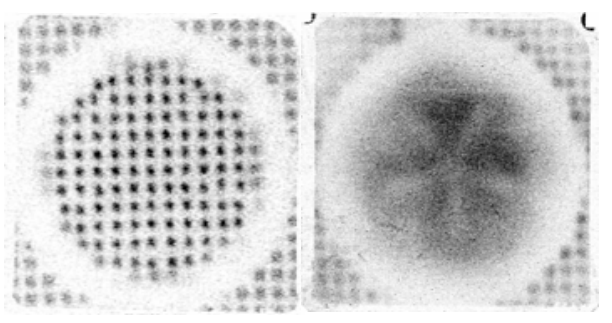

FIG. 14 Images of an Au hohlraum, seen end-on, were made with three types of charged particles and show fields in the hohlraums. In each case, the array of dark spots (high fluence) represent particle beamlets formed by a metal mesh, and the light circle represents the shadow of the $\mathrm{Au}$ hohlraum (low fluence).Images were made with no laser incident and at $0.8 \mathrm{~ns}$ after the laser was incident (the $3-\mathrm{MeV}$ image actually corresponds to $1.0-\mathrm{ns}$, because the lower-energy protons take longer to get from the backlighter to the hohlraum).

To explicitly illustrate the generation and evolution of such an $E$ field, and its effects on proton end-on radiography, a (single) beam laser-foil interaction is simulated (because comprehensive $3 \mathrm{D}$ modeling is currently unavailable). LASNEX calculations indicate that a typical $E$ field has a hemispherical shell structure due to the maximum $\nabla T_{e}\left(\mathbf{E} \propto-\nabla T_{e}\right)$ which occurs around the plasma edge, and is modified by the selfgenerated $B$ field ${ }^{6-8}$. The consequence of side-on radiography (equivalent to end-on radiography of hohlraum) is that the backlighting protons are radially and axially deflected away from the plasma bubble edge as shown in Fig. 15. This feature is more significant (Fig. 15b) due to larger deflections for 3$\mathrm{MeV}$ protons. Quantitative studies indicates that the peak $E$ field on this hohlraum is about $\sim 10^{9} \mathrm{~V} / \mathrm{m}$ due to cavity positive charging, which decays away subsequently ${ }^{45}$. Furthermore, as illustrated in Fig. 16, the measured "asterisk-like" fluence patter (Fig. 15b) is a direct consequence of the uneven distribution of laser beams on a hohlraum wall. Although all incident at 
(a) 15-MeV proton side-on image

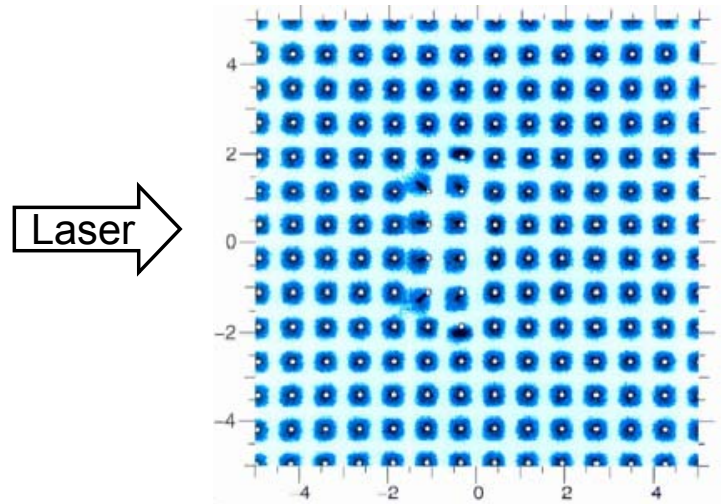

(b) 3-MeV proton side-on image

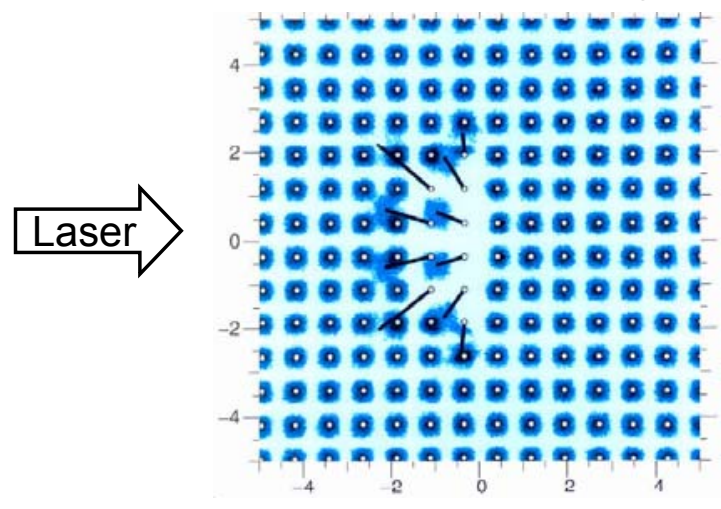

FIG. 15 (Color online) LASNEX+LSP simulated sideon radiography images of $15-\mathrm{MeV} \mathrm{D}^{3} \mathrm{He}$ (a) and 3$\mathrm{MeV}$ DD (b) protons passing through the mesh at different times, indicating that protons radially and axially pushed out from the plasma edge due to the self-generated $E$ fields.

$58.8^{\circ}$ to the normal, the ten laser beams are grouped to form five pairs with $26.8^{\circ}$ between two beams within each pair, but $45.2^{\circ}$ between adjacent pairs (Fig. 16). It is shown that $3-\mathrm{MeV}$ protons scattering off stagnating high-density Au plasmas in the region of the hohlraum center and in the region between two adjacent plasma bubbles are responsible for the formation of such an "asterisk-like" fluence patter ${ }^{45}$. Self-generated $B$ and $E$ fields, on the other hand, will not result in such a striking feature ${ }^{45}$.

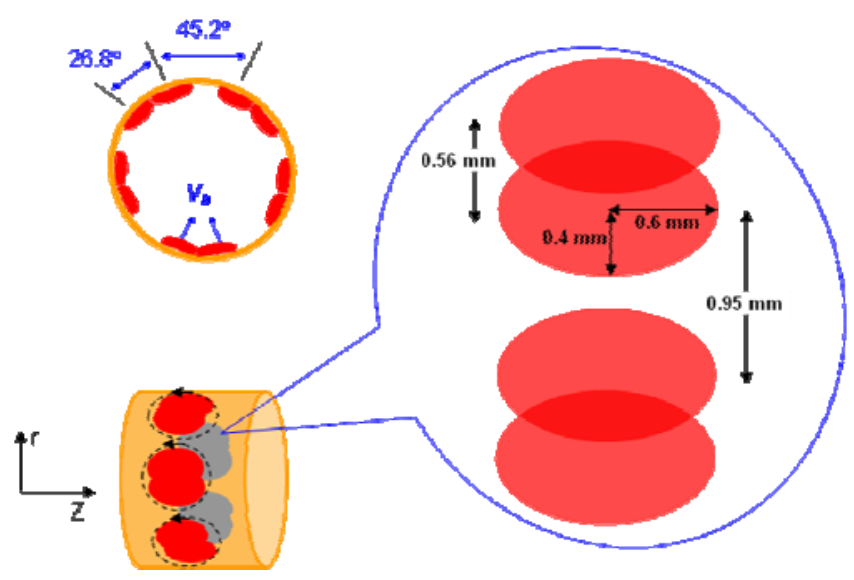

FIG. 16 (Color) Carton to illustrate the formation of the asterisk-like proton fluence shown in the end-on radiograph of Fig. 14.

\section{CONCLUSION}

In summary, we have conducted the first timegated, monoenergetic-proton radiography of selfgenerated electric and magnetic fields associated with laser-foil interactions, imploded ICF capsules and laser-irradiated hohlraums. These experiments resulted in the first observations of several new and important features previously unrealized: first, the observations of the generation, decay dynamics, instabilities and topology change of megagauss $B$ fields on laser-driven planar plastic foil; second, the observation of radial electric fields inside the imploding capsule that are initially directed inward, reversing direction near deceleration onset, and which are likely related to the evolution of the electron pressure gradient; third, the observation of many radial filaments with complex electromagnetic field striations and bifurcations, permeating the entire field of view, and fourth, the observation of $E$ field $\sim 10^{9} \mathrm{~V} / \mathrm{m}$ associated with laserirradiated gold hohlraums. The physics behind and implications of such observed fields have been briefly discussed.

\section{ACKNOWLEDGMENTS}

The work described here was performed in part at the LLE National Laser User's Facility (NLUF), and was supported in part by US DOE (Grant No. DE-FG0303SF22691), LLNL (subcontract Grant No. B504974), and LLE (subcontract Grant No. 412160-001G), the Fusion Science Center at University of Rochester, and GA under DOE (No. DE-AC52-06NA27279). 


\section{REFERENCES}

1.

R. P. Drake High-Energy-Density Physics (Springer, New York 2006).

2

L. Spitzer Physics of Fully Ionized Gases (Intersience Publisher, New York 1962).

3. S. I. Braginskii, Review of Plasma Physics (Consultant Bureau, New York, 1965)

4. M. G. Haines, Phys. Rev. Lett. 78, 254 (1997).

5. M. Borghesi et al., Phys. Rev. Lett. 81, 112 (1998).

6.

C. K. Li et al., Phys. Rev. Lett. 97,135003 (2006).

7. C. K. Li et al., Phys. Rev. Lett. 99,015001 (2007).

8. C. K. Li et al., Phys. Rev. Lett. 99,055001 (2007).

9.

J. Nuckolls et al., Nature 239, 139 (1972).

10. J. D. Lindl, Inertial Confinement Fusion (SpringerVerlag, New York, 1999).

11. S. Atzeni and J. Meyer-Ter-Vehn, The Physics of inertial Confinement Fusion (Claredon Press, Oxford, 2004).

12. C. K. Li et al., Rev. Sci. Instrum. 77, 10E725 (2006)

13. J. M. Soures, et al., Phys. Plasmas 3, 2108 (1996).

14. J. R. Rygg et al., Science 319, 1223 (2008).

15. C. K. Li et al., Phys. Rev. Lett. 100, 225001 (2008).

16. F. H. Séguin et al., Rev. Sci. Instrum. 74,975 (2003).

17. F. H. Séguin et al., Phys. Plasmas.13, 082704 (2006)

18. T. J. Kessler et al., Laser Coherence Control: Technology and Applications (SPIE, Bellingham, WA 1993).

19.

S. Skupsky et al., Phys. Plasmas 6, 2157 (1999).

20. D.D. Meyerhofer et al., Phys. Plasmas 8, 2251 (2001).

21. J. A. Frenje et al., Phys. of Plasmas 11, 2798 (2004)

22. C. K. Li et al., Phys. Plasmas 7, 2578 (2000).

23. A. Mackinnon et al., Rev. Sci. Instrum. 74, 975 (2006)

24. R. D. Petrasso et al., Bull. Am. Phys. Soc. 52, 97 (2007).

25 .

G. B. Zimmerman and W. L. Kruer, Comments Plasma Phys. Control Fusion 2, 51 (1975).

26.

P. D. Nielsen and G. B. Zimmerman, Lawrence Livermore National Laboratory, Livermore, CA, UCRL-53123 (1981).

27.

D. R. Welch et al., Nucl. Instrum. Methods Phys. Res. A 464, 134 (2001).

28. R. P. J. Town et al., Bull. Am. Phys. Soc. 50, 26 (2005).

29.

M. G. Haines, Phys. Rev. Lett. 47, 917 (1981).
30.

J. P. Freidberg, Ideal Magnetohydrodynamics (Plenum Press, New York 1987).

31. S. E. Widnall et al., J. Fluid Mech. 66, 35 (1974).

32.

33. D. Biskamp Magnetic Reconnection in Plasmas M. M. Marinak (private communication). (Cambridge University press, Cambridge 2000).

34. S. Masuda et al., Nature 371, 495 (1994).

35.

T. D. Phan et al., Nature 439, 175 (2006).

36. J. B. Taylor, Rev. Mod. Phys. 58, 741 (1986).

37.

M. A. Yates et al., Phys. Rev. Lett. 49, 1702 (1982).

38. P. A. Sweet, Nuovo Cimento Suppl. 8, Ser.X, 188(1958).

39. E. N. Parker, Astrophys. J. Suppl. Ser. 8177 (1963).

40. M. Tabak et al., Phys. Plasmas 1, 1626 (1994).

41. R. McCrory et al., Phys. Plasmas 15, 055503 (2008).

42. F. H. Séguin et al., paper in preparation

43. J. Deletrezz et al., Phys. Rev. A 36, 3926 (1987).

44. P. A. Amendt et al., Bull. Am. Phys. Soc. 49, 26 (2004).

45. C. K. Li et al., to be submitted to Phys. Rev. Lett. (2008). 